\title{
Abelian $p$-groups with minimal full inertia
}

\author{
Brendan Goldsmith ${ }^{1} \cdot$ Luigi Salce $^{2}$
}

Accepted: 17 December 2020 / Published online: 12 August 2021

(c) The Author(s) 2021

\begin{abstract}
The class of abelian $p$-groups with minimal full inertia, that is, satisfying the property that fully inert subgroups are commensurable with fully invariant subgroups is investigated, as well as the class of groups not satisfying this property; it is known that both the class of direct sums of cyclic groups and that of torsion-complete groups are of the first type. It is proved that groups with "small" endomorphism ring do not satisfy the property and concrete examples of them are provided via Corner's realization theorems. Closure properties with respect to direct sums of the two classes of groups are also studied. A topological condition of the socle and a structural condition of the Jacobson radical of the endomorphism ring of a $p$-group $G$, both of which are satisfied by direct sums of cyclic groups and by torsioncomplete groups, are shown to be independent of the property of having minimal full inertia. The new examples of fully inert subgroups, which are proved not to be commensurable with fully invariant subgroups, are shown not to be uniformly fully inert.
\end{abstract}

Keywords Torsion-complete $p$-group · Direct sum of cyclic $p$-groups $\cdot$ Fully invariant subgroup · Fully inert subgroup · Commensurable subgroups · Minimal full inertia · Endomorphism ring $\cdot$ Pierce decomposition

Mathematics Subject Classification Primary 20K10 $\cdot$ 20K27 · 20K30 · Secondary 20K25

\section{Introduction}

All groups considered in this note are assumed to be abelian. For all unexplained notions we refer to the recent monograph [11] by László Fuchs.

Given an endomorphism $\phi$ of a group $G$, a subgroup $H$ of $G$ is $\phi$-inert if it has finite index in $H+\phi(H)$, and it is fully inert if it is $\phi$-inert for every $\phi \in \operatorname{End}(G)$. The family of fully

\section{Luigi Salce \\ salce@math.unipd.it}

Brendan Goldsmith

brendan.goldsmith@tudublin.ie

1 Technological University Dublin, City Campus, Lower Grangegorman, Dublin 7, D07T795 Dublin, Ireland

2 Dipartimento di Matematica "Tullio Levi-Civita", Università di Padova, Via Trieste 63, 35121 Padova, Italy 
inert subgroups of the $p$-group $G$ is denoted by $\mathcal{I}(G)$; this set will be referred to as the full inertia set of $G$.

A subgroup commensurable with a fully inert subgroup is also fully inert, so, in particular, a subgroup $H$ commensurable with a fully invariant subgroup is fully inert. Following [5] and [6], denote by $\operatorname{Inv}(G)$ the set of fully invariant subgroups of $G$ and by $\operatorname{Inv}(G)$ the set of subgroups of $G$ which are commensurable with fully invariant subgroups.

Then we have the chain of sublattices of the whole lattice $\mathcal{L}(G)$ of subgroups of $G$ :

$$
\mathcal{I} n v(G) \subseteq \mathcal{I} n v^{\sim}(G) \subseteq \mathcal{I}(G) \subseteq \mathcal{L}(G)
$$

In this paper we investigate when the inclusion $\operatorname{In} \tilde{v^{2}}(G) \subseteq \mathcal{I}(G)$ is strict or is an equality for $G$, a reduced $p$-group. Thus, it is useful to introduce the following

Definition 1.1 A $p$-group $G$ is said to have minimal full inertia if the full inertia set $\mathcal{I}(G)$ is equal to $\operatorname{Inv}(G)$.

Results in [15] and [14] indicate that on one hand the group $G$ has minimal full inertia if $\operatorname{End}(G)$ is "big", as in case of unbounded direct sums of cyclic $p$-groups or of unbounded torsion-complete $p$-groups. On the other hand, the group $G$ does not have minimal full inertia, i.e., the strict inclusion $\mathcal{I} n \tilde{v}(G) \subsetneq \mathcal{I}(G)$ holds, when $\operatorname{End}(G)$ is as small as possible, that is, if $\operatorname{End}(G)=J_{p} \cdot 1_{G} \oplus E_{S}(G)$, where $E_{S}(G)$ is the two-sided ideal of the small endomorphisms of $\operatorname{End}(G)$.

There are two ways to look at $\operatorname{End}(G)$, both due to Pierce [20], from which we can argue whether $\operatorname{End}(G)$ is "small" or "big". In fact, given a reduced unbounded p-group $G$, Pierce proved the following two formulas, that we are going to explain:

$$
\operatorname{End}(G)=A \oplus E_{S}(G), \quad \Psi: \operatorname{End}(G) / H(G) \rightarrow \prod_{n} M_{\alpha_{n}}\left(F_{p}\right)
$$

In the left equality in $1.1 A$ is a subring of $\operatorname{End}(G)$ which is the completion in the $p$-adic topology of a free $J_{p}$-module, containing $J_{p} \cdot 1_{G}$, the center of $\operatorname{End}(G)$. We shall call the decomposition $\operatorname{End}(G)=A \oplus E_{S}(G)$ the "Pierce decomposition" of $\operatorname{End}(G)$. Thus, $\operatorname{End}(G)$ is "as small as possible" if in its Pierce decomposition the equality $A=J_{p} \cdot 1_{G}$ holds.

On the right side in 1.1 we have a ring embedding $\Psi$ of the factor $\operatorname{ring} \operatorname{End}(G) / H(G)$, where $H(G)$ is the Pierce radical of $\operatorname{End}(G)$ consisting of the endomorphisms that strictly increase the heights of the elements of the socle $G[p], M_{\alpha_{n}}\left(F_{p}\right)$ is the ring of the $\alpha_{n} \times \alpha_{n}$ matrices over the field with $p$ elements $F_{p}$, and the $\alpha_{n}$ 's are the Ulm-Kaplansky invariants of $G$. It is well known that $\operatorname{Im}(\Psi)$ is a subdirect product of $\prod_{n} M_{\alpha_{n}}\left(F_{p}\right)$, and that $\Psi$ is surjective if and only if $G$ is torsion-complete (see [11, pp. 625-627]). Hence $\operatorname{End}(G)$ is "as big as possible" exactly when $G$ is torsion-complete.

A central role in this context is reserved to semi-standard groups. Recall that a $p$-group $G$ is said to be semi-standard if its Ulm-Kaplansky invariants of finite index $\alpha_{n}(G)$ are finite, and that $G$ is semi-standard if and only if the two-sided ideal of the small endomorphisms $E_{S}(G)$ is contained in the two-sided ideal $E_{0}(G)$ consisting of the endomorphisms $\phi$ such that $\phi(G[p]$ ) is finite (see [22, Proposition 4.1]). The hypothesis that the $p$-group $G$ is semistandard is crucial in our main Theorem 2.4, and it is also assumed in one of the realization theorems by Corner in [4] that, as we will see in Sect. 2, produce examples of groups which do not have minimal full inertia. These theorems extend to a large extent the first construction due to Pierce in [20] of a semi-standard $p$-group $G$ such that $\operatorname{End}(G)=J_{p} \cdot 1_{G} \oplus E_{S}(G)$. This group $G$ furnished the main ingredient in the following theorem proved in [15, Theorem 4.2]. 
Theorem 1.2 If $G$ is a separable p-group of cardinality $2^{\aleph_{0}}$, with semi-standard basic group $B$, such that $\operatorname{End}(G)=J_{p} \cdot 1_{G} \oplus E_{S}(G)$, then the socle $B[p]$ of $B$ is fully inert in $G$ but it is not commensurable with any fully invariant subgroup of $G$.

Thus, Pierce's construction provided the first example of a $p$-group which does not have minimal full inertia. In Sect. 2 of this paper we extend Theorem 1.2 to semi-standard separable p-groups $G$ with endomorphism rings bigger than $J_{p} \cdot 1_{G} \oplus E_{S}(G)$, but still "small". We will see that, in a technical sense, "small" means that the $J_{p}$-algebra $A$ in the Pierce decomposition is the completion of a free $J_{p}$-module of at most countable rank. Thus, Theorem 2.4, the main result of Sect. 2, shows that groups with "small" endomorphism rings do not have minimal full inertia. As recalled above, concrete examples of these groups are obtained via realization theorems by Corner in [4].

In Sect. 3 we consider direct sums of groups that have minimal full inertia, and of groups which do not have minimal full inertia; a nice consequence of the techniques developed here is that one can easily derive a simple proof of the main theorem in [15]: direct sums of cyclic groups have minimal full inertia. We show in Example 3.6 that the class of groups that have minimal full inertia is not closed under taking finite direct sums and that groups which do not have minimal full inertia may have "big" endomorphism ring. We provide also in Proposition 3.9 examples of groups which do not have minimal full inertia obtained via finite direct sums of groups of the same type.

In Sect. 4 we consider two conditions studied by Sands [23] which are satisfied, inter alia, by direct sums of cyclic groups and by torsion-complete groups. The first condition relates to Cauchy sequences of the socle of a $p$-group $G$, and the second concerns a structural condition on the Jacobson radical of $\operatorname{End}(G)$. We show that these conditions are also satisfied by certain groups which do not have minimal full inertia. Thus, these conditions are shown to be independent on the property of having minimal full inertia.

In Sect. 5 we prove that the new examples, furnished in the preceding sections, of fully inert subgroups which fail to be commensurable with fully invariant subgroups are not uniformly fully inert, thus giving further evidence of the likely truth of Conjecture 1.6 in [6], which states that every uniformly fully inert subgroup of an arbitrary group is commensurable with a fully invariant subgroup.

\section{Groups with small endomorphism ring do not have minimal full inertia}

We start by generalizing Theorem 1.2 just noting that its proof can be extended almost verbatim to prove the following

Theorem 2.1 If $G$ is a reduced separable semi-standard p-group such that $\operatorname{End}(G)=$ $J_{p} \cdot 1 \oplus E_{s}(G)$, then, given any pure subgroup $H$ of cardinality $\aleph_{0}$ of $G$, its socles $H\left[p^{k}\right]$ $(k \geq 1)$ belong to $\mathcal{I}(G) \backslash \mathcal{I} n \tilde{v}(G)$.

The hypothesis that $\operatorname{End}(G) / E_{s}(G) \cong J_{p}$ implies that $G$ is not isomorphic to a direct sum of cyclic groups, hence $G$ must be uncountable (see the next Lemma 2.3). An immediate consequence of Theorem 2.1 is the following

Corollary 2.2 Under the hypotheses of the preceding theorem, every subgroup commensurable with a $p^{k}$-socle $(k \geq 1)$ of a pure subgroup of cardinality $\aleph_{0}$ belongs to $\mathcal{I}(G) \backslash \operatorname{In} \tilde{v^{\sim}}(G)$.

The next theorem provides many fully inert subgroups not commensurable with fully invariant subgroups for a wider class of separable $p$-groups, thus extending to a large extent 
Theorem 2.1. First we need the following lemma whose proof utilises an argument from [1, Proposition 2.2].

Lemma 2.3 Let $G$ be an unbounded separable semi-standard p-group with Pierce decomposition of its endomorphism ring $\operatorname{End}(G)=A \oplus E_{s}(G)$. If $A$ is the completion in the p-adic topology of a free $J_{p}$-module $F$ of countable rank, then $G$ is uncountable.

Proof Suppose to the contrary that $G$ is countable. Then, by [11, Theorem 5.3, p. 96], $G$ is a direct sum of cyclic groups, say $G=\bigoplus_{i \in \mathbb{N}} C_{i}$.

Let $\chi: \operatorname{End}(G) \rightarrow \operatorname{Hom}(\mathrm{G}[\mathrm{p}], \mathrm{G})$ be the restriction map sending $\phi \in \operatorname{End}(G)$ into $\phi\left\lceil G[p]\right.$. We claim that $\chi(\operatorname{End}(G))$ has cardinality at least $2^{\aleph_{0}}$. In fact, every element of $\operatorname{Hom}(\mathrm{G}[\mathrm{p}], \mathrm{G})$ can be thought of as an infinite vector of the form $\delta=\left(\delta_{1}, \delta_{2}, \ldots\right)$ where $\delta_{i}$ correspond to homomorphisms from $C_{i}[p]$ into $G$. If we choose $\delta_{i}$ to be either the zero map or the identity map, we can clearly produce $2^{\aleph_{0}}$ homomorphisms in $\operatorname{Hom}(G[p], G)$.

Furthermore, since the zero map and the identity map from $C_{i}[p] \rightarrow G$ both extend trivially to maps from $C_{i} \rightarrow G$, the vector $\delta$ clearly extends to a map $\gamma: G \rightarrow G$ which satisfies $\chi(\gamma)=\delta$. This proves that $\mid \chi\left(\operatorname{End}(G) \mid \geq 2^{\aleph_{0}}\right.$.

However, the image of $A$ under the map $\chi$ is countable, since $\chi(p A)=0$ and $A / p A \cong$ $F / p F \cong\left(J_{p} / p J_{p}\right)^{\left(\aleph_{0}\right)}$. Additionally, as $G$ is semi-standard, every small endomorphism vanishes on a cofinite subgroup $p^{n} G[p]$ of $G[p]$, for some $n$. Since there are only countably many values of $n$, the image $\chi\left(E_{S}(G)\right)$ is countable. Thus, $\chi\left(A \oplus E_{S}(G)\right)$ is also countable, a contradiction. We conclude that $G$ must be uncountable.

Theorem 2.4 Let $G$ be a separable semi-standard p-group such that, in the Pierce decomposition $\operatorname{End}(G)=A \oplus E_{s}(G)$, A is the completion in the p-adic topology of a $J_{p}$-subalgebra $F$ which is a free $J_{p}$-module of at most countable rank. If $H$ is a countable infinite subgroup of $G$, then the higher socles $H^{F}\left[p^{k}\right](k \geq 1)$ of the subgroup $H^{F}=\sum_{\alpha \in F} \alpha(H)$ are fully inert in $G$ but not commensurable with any fully invariant subgroup of $G$.

Proof We give the proof when the rank of $F$ equals $\aleph_{0}$. If the rank is finite, the proof is simpler and it is left to the reader.

First note that $H^{F}$ is $F$-invariant, because $F$ is closed under multiplication. Let $F=$ $\bigoplus_{n \in \mathbb{N}} J_{p} \alpha_{n}$. Clearly $\sum_{n \in \mathbb{N}} \alpha_{n}(H) \leq H^{F}$. Conversely let $\alpha \in F$. Then $\alpha=\sum_{1 \leq i \leq n} \pi_{i} \alpha_{i}$ for suitable $n \in \mathbb{N}$ and $\pi_{i} \in J_{p}$. It follows that $\alpha(H) \leq \sum_{1 \leq i \leq n} \alpha_{i}(H)$, so $H^{F}=$ $\sum_{1 \leq i \leq n} \alpha_{i}(H)$. This equality implies that $H^{F}$ is countable, since each subgroup $\alpha_{i}(H)$ is countable, being an image of $H$; consequently, also $H^{F}\left[p^{k}\right]$ is countable for each $k \geq 1$. As $G$ is uncountable by Lemma $2.3, H^{F}\left[p^{k}\right]$ cannot be commensurable with a nonzero fully invariant subgroup of $G$, since these subgroups are also uncountable, as proved in [15, Theorem 4.2].

To prove that $H^{F}\left[p^{k}\right]$ is fully inert, we must show that, given any endomorphism $\phi \in$ $\operatorname{End}(G),\left(H^{F}\left[p^{k}\right]+\phi\left(H^{F}\left[p^{k}\right]\right)\right) / H^{F}\left[p^{k}\right]$ is finite. Let $\phi=\alpha+\theta$, with $\alpha \in A$ and $\theta \in E_{S}(G)$. As $A$ is the completion of $F$, for each $n \in \mathbb{N}$ there exists a $\phi_{n} \in F$ such that $\alpha-\phi_{n} \in p^{n} A$. Then we have $\alpha\left(H^{F}\left[p^{k}\right]\right)=\left(\alpha-\phi_{k}\right)\left(H^{F}\left[p^{k}\right]\right)+\phi_{k}\left(H^{F}\left[p^{k}\right]\right)$. But $\left(\alpha-\phi_{k}\right)\left(H^{A}\left[p^{k}\right]\right)=0$ because $\alpha-\phi_{k} \in p^{k} A$, and $\phi_{k}\left(H^{F}\left[p^{k}\right]\right) \leq H^{F}\left[p^{k}\right]$, because $H^{F}\left[p^{k}\right]$ is $F$-invariant. Therefore it is enough to prove that $\left.\theta\left(H^{F}\left[p^{k}\right]\right)\right)$ is finite.

Since $H^{F}$ is countable, a classical result by Szele (see [11, Theorem 1.5, p. 151]) ensures that there exists a countable pure subgroup of $G, C$ say, with $H^{F} \leq C$. Now $C$ is countable and separable and hence it is a direct sum of cyclic groups; we write $C=\bigoplus i \geq 1 C_{i}$ where each $C_{i}$ is a direct sum of cyclic groups of order $p^{i}$ (possibly zero). Thus, each $C_{i}$ is a 
bounded pure subgroup of $C$ and hence is a direct summand of $G$. Since, by hypothesis, $G$ is semi-standard, each $C_{i}$ is of finite rank.

Now $H^{F}\left[p^{k}\right] \leq C\left[p^{k}\right]=\left(C_{1} \oplus \cdots \oplus C_{k}\right) \oplus p C_{k+1} \oplus p^{2} C_{k+2} \oplus \cdots$ and since $\theta$ is small, there exists a positive integer $N$ such that $\theta\left(p^{N} G\left[p^{k}\right]\right)=0$. Hence $\theta\left(p^{N} C_{N+k} \oplus\right.$ $\left.p^{N+1} C_{N+k+1} \oplus \cdots\right)=0$. Thus, $\theta\left(H^{F}\left[p^{k}\right]\right) \leq \theta\left(C_{1} \oplus \cdots \oplus C_{k} \oplus p C_{k+1} \oplus \cdots \oplus\right.$ $\left.p^{N-1} C_{N+k-1}\right)$ and this latter term is finite since it is a homomorphic image of a finite direct sum of finite groups.

In the next remark and in the following we deal with the algebraic entropy of endomorphisms of $p$-groups. For an illustration of the notion of algebraic entropy and its properties we refer to [8] and to our survey paper [13]. We just recall here that the algebraic entropy of an endomorphism $\phi$ of an abelian group $G$, denoted by ent $(\phi)$, is an invariant (actually, a length function on the category of $\mathbb{Z}[X]$-modules), which measures by means of nonnegative real numbers and the symbol $\infty$ the behaviour of the discrete dynamical system obtained by the powers of $\phi$.

Remark 2.5 If a $J_{p}$-algebra $A$ is a free $J_{p}$-module of finite rank, then it is integral over $J_{p}$. This implies (see [8, Proposition 2.4]) that every endomorphism $\phi$ of a semi-standard p-group $G$ such that $\operatorname{End}(G)=A \oplus E_{S}(G)$ has algebraic entropy ent $(\phi)$ equal to 0 . In fact, ent $(\phi)=0$ is equivalent to the fact the $\phi$ is pointwise integral over $G$, which means that for every $x \in G$ there exists a monic polynomial $g(X) \in J_{p}[X]$ depending on $x$ such that $g(\phi)(x)=0$. Looking at Theorem 2.4, one could conjecture that the strict inclusion $\mathcal{I} n v^{\sim}(G) \subsetneq \mathcal{I}(G)$ is related to the property of $G$ of having the total entropy $\operatorname{ent}(G)=0$ (i.e., $\operatorname{ent}(\phi)=0$ for all $\phi \in \operatorname{End}(G))$. The following discussion will show that this conjecture is wrong; see Proposition 2.8 below.

At this point, a natural question arises. Can we find separable semi-standard $p$-groups $G$ satisfying the hypotheses of Theorem 2.4 ?

The answer comes from the realization theorems proved by Corner in his outstanding paper on endomorphism rings of separable $p$-groups. One of these theorems ( $[4$, Theorem 4.1]) is the following.

Theorem 2.6 [Corner] Let A be a $J_{p}$-algebra which is the completion of a free $J_{p}$-module of countable rank. If A satisfies the following condition:

(*) there exists a descending sequence of right ideals $A \geq A_{1} \geq A_{2} \geq \cdots \geq A_{n} \geq \cdots$

such that $A_{i} / A_{i+1}$ is a free $J_{p}$-module of finite rank for each $i$ and $p A=\cap_{i}\left(p A+A_{i}\right)$, then there exists a separable semi-standard p-group $G$ such that $\operatorname{End}(G)=A \oplus E_{S}(G)$.

In [8, Example 5.12] a $J_{p}$-algebra $A$ integral over $J_{p}$ was constructed using the Nagata idealization satisfying the hypothesis of Corner's Theorem 2.6. The $p$-group $G$ obtained via Theorem 2.6 such that $\operatorname{End}(G)=A \oplus E_{S}(G)$ had total entropy ent $(G)=0$, because of the integrality of $A$ over $J_{p}$.

Another powerful theorem in Corner's paper ( [4, Theorem 2.1]) is the following

Theorem 2.7 [Corner] Let $\bar{B}$ be a torsion-complete p-group with an unbounded basic subgroup $B$ of cardinality at most $2^{\aleph_{0}}$, and let $\Phi$ be a separable closed subring of $\operatorname{End}(\bar{B})$ that leaves $B$ invariant and satisfies the condition

(C) if $\phi \in \Phi$ and $\phi\left(p^{n} \bar{B}[p]\right)=0$ for some $n$, then $\phi \in p \Phi$.

Then there exists a family $G_{\sigma}(\sigma \in \Sigma)$ of $2^{2{ }^{\aleph_{0}}}$ pure subgroups of $\bar{B}$ containing $B$ such that

(a) for each $\sigma \in \Sigma$, $\operatorname{End}\left(G_{\sigma}\right)=\Phi \oplus E_{S}\left(G_{\sigma}\right)$;

(b) for distinct $\sigma, \tau \in \Sigma$, every homomorphism $G_{\sigma} \rightarrow G_{\tau}$ is small. 
Using this theorem, in [8, Theorem 4.4] it was proved that there exist $2^{2^{\aleph_{0}}}$ non-isomorphic groups $G$ with standard basic subgroup $B=\bigoplus_{n \in N} \mathbb{Z}\left(p^{n}\right)$ and contained in the torsioncompletion $\bar{B}$ of $B$, such that $\operatorname{End}(G)=A \oplus E_{S}(G)$, where $A$ is isomorphic to the $p$-adic completion of the polynomial ring $J_{p}[X]$. Furthermore, if $\omega$ denotes the endomorphism of $G$ corresponding to the indeterminate $X$, then ent $(\omega)=\infty$. This depends on the fact that $\omega$ is not only nonalgebraic over $J_{p}$, but it fails also to be pointwise integral over $G$.

To sum up, we can answer the above question as follows.

Using Corner's realization theorems we can construct separable semi-standard $p$-groups $G$ satisfying the hypotheses of our Theorem 2.4 , with the $J_{p}$-algebra $A$ either integral over $J_{p}$, or neither integral over $J_{p}$ nor pointwise integral over $G$. All these groups do not have minimal full inertia. This shows that the proper inclusion $\mathcal{I} n v^{\sim}(G) \subsetneq \mathcal{I}(G)$ is independent of the vanishing of the total entropy of $G$. In conclusion, we have seen that

Proposition 2.8 There exists separable semi-standard p-groups $G$ which do not have minimal full inertia such that $\operatorname{ent}(G)=0$ and also such that $\operatorname{ent}(G)=\infty$.

\section{Minimal full inertia for direct sums of p-groups}

In this section we investigate direct sums of groups which have minimal full inertia and of groups which do not have minimal full inertia.

We are interested in identifying fully inert subgroups up to commensurability. The following lemma was proved in [2, Lemma 7] using an idea in [15, Lemma 3.3].

Lemma 3.1 [Chekhlov] If $G=\bigoplus_{i \in I} G_{i}$ is a direct sum of arbitrary groups, $\pi_{i}: G \rightarrow G_{i}$ are the canonical projections, and $H$ is a fully inert subgroup, then $H$ is commensurable with $\bigoplus_{i \in I} \pi_{i}(H)$.

Thus, from now on, when dealing with fully inert subgroups $H$ of a direct sum of cyclic groups $\bigoplus_{i \in I} G_{i}$, we will assume that $H=\bigoplus_{i \in I} H_{i}$, with $H_{i} \leq G_{i}$ for all $i$. This situation is expressed by saying that $H$ is a box-like subgroup of $G$ in [7], where this terminology was introduced and this notion was used for direct sums of divisible groups; it was used also more recently in [3], [6] and [14].

The following result concerning box-like subgroups was proved in [2] and [3] and is based on results in [2] and [14]. For the convenience of the reader we present a proof of part (b); this is a very slight modification of that given in [3, Lemma 2.2].

Lemma 3.2 [Chekhlov, Chekhlov-Danchev-Goldsmith] Let $G=\bigoplus_{i \in I} G_{i}$ be a direct sum of arbitrary groups, $\pi_{i}: G \rightarrow G_{i}$ the canonical projections, and $H=\bigoplus_{i \in I} H_{i}$ a box-like subgroup. If $H$ is fully inert in $G$, then the following conditions hold:

(a) $H_{i}$ is fully inert in $G_{i}$ for all $i$ and is fully invariant in $G_{i}$ for almost all $i$;

(b) if I is infinite, then there exists a finite subset $I_{0}$ of I such that $\bigoplus_{i \in I \backslash I_{0}} H_{i}$ is fully invariant in $\bigoplus_{i \in I \backslash I_{0}} G_{i}$.

Proof Proof of part (b): By point (a), there exists a cofinite subset $I_{1}$ of $I$ such that $H_{i}$ is fully invariant in $G_{i}$ for all $i \in I_{1}$. We will prove that there exists a cofinite set of indices $I_{0}$ contained in $I_{1}$ such that the whole direct sum $\bigoplus_{i \in I_{0}} H_{i}$ is fully invariant in $\bigoplus_{i \in I_{0}} G_{i}$.

Consider the set

$$
S=\left\{j \in I_{1} \mid \text { there exist } i \in I_{1} \text { and } \phi_{i j}: G_{i} \rightarrow G_{j} \text { such that } \phi_{i j}\left(H_{i}\right) \not \leq H_{j}\right\} .
$$


If the set $S$ is infinite, for each $j \in S$ choose an index $i \in I_{1}$ and a map $\phi_{i j}: G_{i} \rightarrow G_{j}$ such that $\phi_{i j}\left(H_{i}\right) \not \leq H_{j}$ and define the endomorphism $\psi$ of $\bigoplus_{i \in I} G_{i}$ by setting $\psi \uparrow G_{i}=\phi_{i j}$ for each one of these chosen maps $\phi_{i j}$, and extend trivially on the remaining direct summands of $G$. By the definition of $S$, for each $j \in S$ and the corresponding map $\phi_{i j}$, there exists an element $x_{j} \in G_{j}$ such that $x_{j} \in \phi_{i j}\left(H_{i}\right) \backslash H_{j}$. Now the quotient group $(\psi(H)+H) / H$ contains the cosets $x_{j}+H$ for each one of these indices $j$. We claim that these cosets are all distinct; in fact, if $x_{j}+H=x_{t}+H$ for some $j \neq t$, then $x_{j}-x_{t} \in H$, so that $\pi_{j}\left(x_{j}-x_{t}\right)=x_{j} \in \pi_{j}(H)=H_{j}$, absurd. Therefore the quotient $(\psi(H)+H) / H$ is infinite, and this contradicts the full inertia of $H$. We derive that the set $S$ is finite. Removing this finite set from $I_{1}$, we obtain a cofinite subset $I_{0}$ of $I$ such that for all $j \in I_{0}$ and for all $i \in I_{1}$-and a fortiori for all $i \in I_{0}$-it happens that $\phi\left(H_{i}\right) \subseteq H_{j}$ for all maps $\phi: G_{i} \rightarrow G_{j}$. Since $\operatorname{End}\left(\bigoplus_{i \in I} G_{i}\right) \cong \prod_{i} \operatorname{Hom}\left(\mathrm{G}_{\mathrm{i}}, \bigoplus_{\mathrm{j} \in \mathrm{I}} \mathrm{G}_{\mathrm{j}}\right) \leq \prod_{\mathrm{i}, \mathrm{j}} \operatorname{Hom}\left(\mathrm{G}_{\mathrm{i}}, \mathrm{G}_{\mathrm{j}}\right)$, it follows that $\bigoplus_{i \in I_{0}} H_{i}$ is fully invariant in $\bigoplus_{i \in I_{0}} G_{i}$.

From Lemmas 3.1 and 3.2 it is possible to deduce a very simple proof of the main theorem in [15], which states, in our terminology, that direct sums of cyclic $p$-groups have minimal full inertia. The proof was very long and elaborate, split into the bounded and the unbounded cases, with many intermediate results of independent interest. Even allowing for the fact that detailed proofs of these lemmas require careful arguments, the total effort to prove the theorem in this way is much lower than that required in [15] and as such, this represents a substantial improvement on the original solution. We illustrate this by including the proof as a corollary to Lemma 3.2.

\section{Corollary 3.3 If $G$ is a direct sum of cyclic p-groups, then $G$ has minimal full inertia.}

Proof Suppose that $G=\bigoplus_{i \in I} C_{i}$ where each $C_{i}$ is a cyclic $p$-group, and let $H$ be an arbitrary fully inert subgroup of $G$. As observed above, we may assume that $H=\bigoplus_{i \in I} H_{i}$ is a box-like subgroup of $G$. Then it follows from Lemma 3.2 that there is a finite subset $I_{0}$ of $I$ such that $M=\bigoplus_{i \in I \backslash I_{0}} H_{i}$ is fully invariant in $G_{0}=\bigoplus_{i \in I \backslash I_{0}} C_{i}$; setting $F_{0}=\bigoplus_{i \in I_{0}} C_{i}$, we have that $H \sim F \oplus M$ where $F=\bigoplus_{i \in I_{0}} H_{i}$ is a subgroup of the finite group $F_{0}$. Now it is well known - see for example [15, Lemma 1.5] - that there is a subgroup $F_{1}$ of $F_{0}$ such that $F_{1} \oplus M$ is fully invariant in $G$. Since $F_{1}$ is also finite, we have $H \sim F \oplus M \sim F_{1} \oplus M$ and the latter is fully invariant in $G$. Since $H$ was an arbitrary fully inert subgroup of $G, G$ has minimal full inertia.

The result in Corollary 3.3 has been extended to arbitrary totally projective $p$-groups by Patrick Keef in the remarkable paper [18].

We consider now direct sums of a fixed group $G$ which has minimal full inertia, under the additional hypothesis that $G$ is fully transitive; recall that a separable $p$-group is fully transitive. We start with the case of a finite direct sum.

Lemma 3.4 If $X=G_{1} \oplus G_{2} \oplus \cdots \oplus G_{n}$, where $G_{i}=G$ for all $i$, and $G$ is an unbounded fully transitive p-group which has minimal full inertia, then $X$ also has minimal full inertia.

Proof Let $H$ be a fully inert subgroup of $X$. Then $H$ is commensurable with $H_{1} \oplus H_{2} \oplus$ $\cdots \oplus H_{n}$, where $H_{i}=H \cap G_{i}$, which is still fully inert. Then Lemma 3.2 (a) and the fact that $G$ is fully transitive ensure that each $H_{i}$ is commensurable with $G\left(\mathbf{u}_{\mathbf{i}}\right)(i=1,2, \ldots, n)$, for suitable increasing sequences of ordinals or symbols $\infty$. So $H$ is commensurable with $G\left(\mathbf{u}_{1}\right) \oplus G\left(\mathbf{u}_{2}\right) \oplus \cdots \oplus G\left(\mathbf{u}_{\mathbf{n}}\right)$ and this subgroup is still fully inert. Then [2, Proposition 1] ensures that, for every homomorphism $\alpha: G_{i} \rightarrow G_{j}$, with $i \neq j$, the quotient $\left(\alpha\left(G\left(\mathbf{u}_{\mathbf{i}}\right)\right)+\right.$ $\left.G\left(\mathbf{u}_{\mathbf{j}}\right)\right) / G\left(\mathbf{u}_{\mathbf{j}}\right)$ is finite. In particular, if $\alpha=1_{G}$, we have

$$
\left(G\left(\mathbf{u}_{\mathbf{i}}\right)+G\left(\mathbf{u}_{\mathbf{j}}\right)\right) / G\left(\mathbf{u}_{\mathbf{j}}\right)=G\left(\mathbf{u}_{\mathbf{i}} \cap \mathbf{u}_{\mathbf{j}}\right) / G\left(\mathbf{u}_{\mathbf{j}}\right) .
$$


Clearly this implies that each subgroup $G\left(\mathbf{u}_{\mathbf{i}}\right)$ is commensurable with $G\left(\mathbf{u}_{\mathbf{1}} \cap \mathbf{u}_{\mathbf{2}} \cap \cdots \cap \mathbf{u}_{\mathbf{n}}\right)$. In conclusion, setting $\mathbf{w}=\mathbf{u}_{\mathbf{1}} \cap \mathbf{u}_{\mathbf{2}} \cap \cdots \cap \mathbf{u}_{\mathbf{n}}$, we have that $H$ is commensurable with $G(\mathbf{w}) \oplus G(\mathbf{w}) \oplus \cdots \oplus G(\mathbf{w})=X(\mathbf{w})$, therefore $X$ has minimal full inertia.

We extend now Lemma 3.4 to infinite direct sums of a group with minimal full inertia.

Theorem 3.5 If $X=\bigoplus_{i \in I} G_{i}$, where each $G_{i}$ is isomorphic to a fixed unbounded fully transitive p-group which has minimal full inertia, then $X$ has minimal full inertia.

Proof Let $H$ be a fully inert subgroup of $X$ and let $\pi_{i}: X \rightarrow G_{i}$ be the canonical projections. From Lemma 3.2 we get that $H$ is commensurable with $\bigoplus_{i \in I} \pi_{i}(H)$, with $\pi_{i}(H)$ fully inert in $G_{i}$ for all $i \in I$; furthermore, there exists a finite subset $I_{0}$ of $I$ such that $X=C \oplus A$ where $C=\bigoplus_{i \in I_{0}} G_{i}$ and $A=\bigoplus_{i \in I \backslash I_{0}} G_{i}$ and $\bigoplus_{i \in I \backslash I_{0}} H_{i}$ is actually fully invariant in $A$. Note that $C$ has minimal full inertia, by Lemma 3.4, and $\bigoplus_{i \in I_{0}} H_{i}$ is fully inert in $C$.

Since $A$ is fully transitive, $\bigoplus_{i \in I \backslash I_{0}} H_{i}$ can be expressed in the form $A(\mathbf{v})$ for some suitable $U$-sequence v. Lemma 3.4 ensures that $\bigoplus_{i \in I_{0}} H_{i}$ is commensurable with a fully invariant subgroup of $C$ of the form $C(\mathbf{u})$ for a $U$-sequence $\mathbf{u}$. Hence we have that $H$ is commensurable with $C(\mathbf{u}) \oplus A(\mathbf{v})$, thus $C(\mathbf{u}) \oplus A(\mathbf{v})$ is fully inert in $X$, and these summands are fully invariant in $C$ and $A$, respectively.

We are now in a position to apply Lemma 3.2. For any homomorphism $\gamma: C \rightarrow A$ the quotient $(\gamma(C(\mathbf{u}))+A(\mathbf{v})) / A(\mathbf{v})$ is finite. In particular, choosing $\gamma$ to be an isomorphism from $C$ onto a direct summand $Z$ of $A$, say $A=Z \oplus Y$, we have that $\gamma(C(\mathbf{u}))=Z(\mathbf{u})$. A simple argument shows that $Z(\mathbf{v})$ is commensurable with $Z(\mathbf{u} \cap \mathbf{v})$.

Similarly, for any homomorphism $\delta: A \rightarrow C$, we have $(\delta(A(\mathbf{v})+C(\mathbf{u})) / C(\mathbf{u})$ is finite. Now $A=Z \oplus Y$ and choosing $\delta$ in such a way that $\delta(Z)=C$, we have $A(\mathbf{v})=Z(\mathbf{v}) \oplus Y(\mathbf{v})$ and $\delta(Z(\mathbf{v}))=C(\mathbf{v})$. So we have that $(C(\mathbf{v})+C(\mathbf{u})) / C(\mathbf{u})=C(\mathbf{u} \cap \mathbf{v}) / C(\mathbf{u})$ is finite; hence $C(\mathbf{u})$ is commensurable with $C(\mathbf{u} \cap \mathbf{v})$. But $C(\mathbf{u} \cap \mathbf{v}) / C(\mathbf{v}) \cong Z(\mathbf{u} \cap \mathbf{v}) / Z(\mathbf{v})$ is finite, hence also $C(\mathbf{v})$ is commensurable with $C(\mathbf{u} \cap \mathbf{v})$. It now follows immediately that $H$ is commensurable with $C(\mathbf{v}) \oplus A(\mathbf{v})=X(\mathbf{v})$. Thus, $X$ has minimal full inertia, as required.

Theorem 3.5 applies in particular when the groups $G_{i}$ are isomorphic to a fixed unbounded torsion-complete group.

The next example shows that the class of groups which have minimal full inertia is not closed under taking finite direct sums.

Example 3.6 Let $G=B_{1} \oplus \bar{B}_{2}$, where $B_{1}$ is an unbounded direct sum of cyclic $p$-groups and $\bar{B}_{2}$ is an unbounded semi-standard torsion-complete group. We claim that $G$ does not have minimal full inertia.

In fact, the subgroup $H=\{0\} \oplus \bar{B}_{2}[p]$ is fully inert, by Proposition 3.1; the only nonobvious condition to be verified is that, for every homomorphism $\alpha: \bar{B}_{2} \rightarrow B_{1}$, $\left(\alpha \bar{B}_{2}[p]+\{0\}\right) /\{0\}=\alpha \bar{B}_{2}[p]$ is finite. But $\alpha$ is small by a well-known result of Megibben (see [11, Exercise 14, p. 317]), hence $\alpha\left(p^{N} \bar{B}_{2}[p]\right)=0$ for a suitable $N \in \mathbb{N}$. As $\bar{B}_{2}$ is semi-standard, it follows that $\alpha \bar{B}_{2}[p]$ is finite, so $H$ is fully inert. On the other hand, $H$ is not commensurable with a fully invariant subgroup of $G$; as $G$ is fully transitive, such a subgroup is of the form $G(\mathbf{u})$ for some sequence $\mathbf{u}$. But $G(\mathbf{u})=B_{1}(\mathbf{u}) \oplus \bar{B}_{2}(\mathbf{u})$, and $B_{1}(\mathbf{u})$ is never finite, unless $B_{1}(\mathbf{u})=0$, equivalently, $\mathbf{u}=(\infty, \infty, \ldots)$, in which case also $\bar{B}_{2}(\mathbf{u})=0$.

Some comments on Example 3.6 are in order. First, in place of $B_{1}$ we could use any unbounded separable $p$-group not containing an unbounded torsion-complete group. Furthermore, if either $B_{1}$ or $\bar{B}_{2}$ is bounded, then $G$ has minimal full inertia, since it becomes 
either torsion-complete or a direct sum of cyclic groups. Example 3.6 is also interesting, since it shows that also groups with "big" endomorphism ring may fail to have minimal full inertia and that the property of being semi-standard is independent of the property of having minimal full inertia. Finally, Example 3.6 may be generalized by the following proposition, which enables us to produce many $p$-groups which do not have minimal full inertia.

Proposition 3.7 If $G=A \oplus C$ is a separable p-group which is the direct sum of two unbounded groups $A$ and $C$, such that $A$ is semi-standard and every homomorphism from $A$ to $C$ is small, then $G$ does not have minimal full inertia.

Proof Let $H=A[p] \oplus\{0\}$. Then the same argument as used in Example 3.6 shows that $H$ is fully inert in $G$. Suppose to the contrary that $H$ is commensurable with a fully invariant subgroup of $G$, say, $G(\mathbf{u})=A(\mathbf{u}) \oplus C(\mathbf{u})$ for some U-sequence $\mathbf{u}=\left(u_{0}, u_{1}, \ldots\right)$; since $G$ is separable, $u_{0}$ is a nonnegative integer. Then $C(\mathbf{u})$ must be commensurable with $\{0\}$, that is, it must be finite. However, as $C$ is unbounded, this is impossible, so we get the desired contradiction.

Lemma 3.2 makes it possible to construct more examples of $p$-groups which fail to have minimal full inertia using direct sums of these groups. Selecting finitely many groups obtained in the above-mentioned [8, Theorem 4.4], which uses Corner's Theorem [4, Theorem 2.1], we get the following

Example 3.8 Let $G_{1}, G_{2}, \ldots, G_{n}$ be nonisomorphic groups such that $\operatorname{End}\left(G_{i}\right)=A \oplus$ $E_{s}\left(G_{i}\right)$, where $A$ is isomorphic to the $p$-adic completion of the polynomial ring $J_{p}[X]$ and every homomorphism $G_{i} \rightarrow G_{j}$ is small for $i \neq j$. We claim that $G=\bigoplus_{1 \leq i \leq n} G_{i}$ does not have minimal full inertia.

In fact, choose arbitrary countable subgroups $H_{i}$ in $G_{i}$ for all $i$. Using the notation of Theorem 2.4, set $K_{i}=H_{i}^{J_{p}^{[}[X]}[p]$ for all $i$. We claim that the subgroup $K=\bigoplus_{1 \leq i \leq n} K_{i}$ is fully inert in $G$ but not commensurable with any fully invariant subgroup, so that $\mathcal{I}_{n} \tilde{v}^{\sim}(G) \subsetneq$ $\mathcal{I}(G)$. The fact that $K$ is fully inert follows from Lemma 3.2, because all the subgroups $K_{i}$ are fully inert in $G_{i}$, by Theorem 2.4, and the fact that every homomorphism $\alpha_{i j}: G_{i} \rightarrow G_{j}$ is small for $i \neq j$ implies that $\alpha_{i j} K_{i}$ is finite; therefore $\left(\alpha_{i j} K_{i}+K_{j}\right) / K_{j}$ is finite too. Finally, $K$ is not commensurable with a fully invariant subgroup of $G$ because such a subgroup is uncountable and $K$ is countable.

Again, using the results and the notation of [8, Theorem 4.4], we can generalize the preceding example to the following

Proposition 3.9 If $G_{\sigma}(\sigma \in \Sigma)$ is a family of $2^{2^{\aleph_{0}}}$ separable groups with basic subgroup $B=\bigoplus_{n} \mathbb{Z}\left(p^{n}\right)$ such that $\operatorname{End}\left(G_{\sigma}\right)=A \oplus E_{s}\left(G_{\sigma}\right)$, where $A$ is isomorphic to the $p$-adic completion of the polynomial ring $J_{p}[X]$, and every homomorphism $G_{\sigma} \rightarrow G_{\tau}$ is small for $\sigma \neq \tau$, then $G=\bigoplus_{\sigma} G_{\sigma}$ does not have minimal full inertia.

Proof Fix a $\tau \in \Sigma$ and take a countable subgroup $H_{\tau}$ in $G_{\tau}$. Using the notation of Theorem 2.4, set $K_{\tau}=H_{\tau}^{J_{p}[X]}[p]$. We claim that the subgroup $K=\bigoplus_{\sigma \in \Sigma} K_{\sigma}$, where $K_{\sigma}=0$ for all $\sigma \neq \tau$, is fully inert in $G$ but not commensurable with any fully invariant subgroup, so that $\mathcal{I} n \tilde{v}(G) \subsetneq \mathcal{I}(G)$. The fact that $K$ is fully inert follows from Lemma 3.2, since all the subgroups $K_{\sigma}$ are trivially fully invariant in $G_{\sigma}$, except $K_{\tau}$ which is fully inert in $G_{\tau}$, and since every homomorphism $\alpha_{\tau \sigma}: G_{\tau} \rightarrow G_{\sigma}$ small implies that $\alpha_{\tau \sigma} K_{\tau}$ is finite; therefore $\left(\alpha_{\tau \sigma} K_{\tau}+K_{\sigma}\right) / K_{\sigma}$ is finite too. Furthermore, condition (c) of Lemma 3.2 is satisfied trivially for $I_{0}=\{\sigma\}$. Finally, $K$ is not commensurable with a nonzero fully invariant subgroup of $G$, because such a subgroup is uncountable and $K$ is countable. 


\section{Trying to enlarge the family of $p$-groups with minimal full inertia}

Up to now the only concrete examples of separable $p$-groups with minimal full inertia at disposal are the direct sums of cyclic groups, the torsion-complete groups and the direct sums of a fixed torsion-complete group. We would like to enlarge the family of groups with minimal full inertia, even if we guess that a characterization of these groups via structural properties of the groups themselves, or of their endomorphism ring, is hopeless.

To justify this idea, we consider properties of $p$-groups $G$ and of their endomorphism ring $\operatorname{End}(G)$ investigated by Arthur D. Sands in [23]. Sands calls sufficiently projective a separable $p$-group $G$ such that every countable subset is contained in a direct summand of $G$ that is a direct sum of cyclic groups. Sufficiently projective $p$-groups are called also $\aleph_{1}$-separable (or $\omega_{1}$-separable) by other authors, and have been investigated also using settheoretic techniques (see [17], [10, Chapter VIII], [19] and [12]). Clearly direct sums of cyclic groups are sufficiently projective and a torsion-complete group is sufficiently projective exactly if it is bounded. Hill gave in [16] an example of a sufficiently projective group which fails to be a direct sum of cyclics. Sands notes in [23] that sufficiently projective groups and torsion-complete groups satisfy the following technical condition:

(C) given any Cauchy sequence $\left\{g_{i}\right\}_{i \in \mathbb{N}}$ in $G[p]$ which is not convergent in $G$, there exists a direct sum of cyclic groups $H$ and a homomorphism $\alpha: G \rightarrow H$ such that $\left\{\alpha\left(g_{i}\right)\right\}_{i \in \mathbb{N}}$ is Cauchy but not convergent in $H$.

Warning: the preceding condition $(\mathrm{C})$ introduced by Sands is not to be confused with condition $(C)$ in Corner's Theorem 2.7 .

One of the main results in Sands's paper, [23, Theorem 5], is that, if a group $G$ satisfies condition (C), then the Jacobson radical $J(\operatorname{End}(G))$ of $\operatorname{End}(G)$ equals $H(G) \cap C(G)$, where $H(G)$ is the Pierce radical mentioned in the Introduction, and $C(G)$ the ideal of $\operatorname{End}(G)$ consisting of those endomorphisms which send Cauchy sequences of the socle $G[p]$ into convergent sequences. In general the inclusion $H(G) \cap C(G) \subseteq J(\operatorname{End}(G))$ holds, and Dugas gave in [9] an example of a $p$-group for which the strict inclusion holds.

Thus, we may consider this condition (C) for a $p$-group $G$ and the condition $J(\operatorname{End}(G))=$ $H(G) \cap C(G)$ for its endomorphism ring, and we may ask whether groups satisfying these conditions have minimal full inertia. The next result shows that having minimal full inertia is independent of both these conditions.

Proposition 4.1 There exist separable p-groups $G$ satisfying Sands's condition $(C)$, or such that $J(\operatorname{End}(G))=H(G) \cap C(G)$, which have minimal full inertia and also which do not have minimal full inertia.

Proof Concerning condition (C), on one hand, we have seen in Example 3.6 that the group $G=B_{1} \oplus \bar{B}_{2}$, where $B_{1}$ is an unbounded direct sum of cyclic $p$-groups and $\bar{B}_{2}$ is an unbounded semi-standard torsion-complete group, does not have minimal full inertia. It is quite obvious that this group $G$ satisfies condition (C). On the other hand, both direct sums of cyclic groups and torsion-complete groups satisfy condition (C) and have minimal full inertia.

Concerning the condition $J(\operatorname{End}(G))=H(G) \cap C(G)$, on one hand, the results by Sands show that sufficiently projective groups, and in particular direct sums of cyclic groups, and torsion-complete groups satisfy this equality; all these groups have minimal full inertia. On the other hand, Sands noted in [23] that the Pierce group $G$ such that $\operatorname{End}(G)=J_{p} \cdot 1_{G} \oplus E_{S}(G)$ also has this property; this group $G$ was the first example of a group which has not minimal full inertia. 
We conclude this section with the following still unanswered question: do sufficiently projective groups have minimal full inertia?

\section{The uniform full inertia set}

Before our present investigation, there was only one available example of a fully inert subgroup of a $p$-group not commensurable with a fully invariant subgroup, namely, the socle $B[p]$ of a basic subgroup $B$ of the separable $p$-group $G$ provided by Theorem 1.2, which uses Pierce's construction of $G$ such that $\operatorname{End}(G)=J_{p} \cdot 1_{G} \oplus E_{S}(G)$ (see [15, Theorem 4.2]).

In [6, Proposition 6.2] it was proved that the socle $B[p]$ is not uniformly fully inert, that is, the cardinalities of the quotients $(\phi B[p]+B[p]) / B[p]$, ranging $\phi$ in $\operatorname{End}(G)$, do not have a uniform bound $k \in \mathbb{N}$.

This fact, together with many other exhibited examples for different families of abelian groups, tempted the authors of [6] to formulate the conjecture (already presented in [5]) that every uniformly fully inert subgroup of a group $G$ is commensurable with a fully invariant subgroup. Following [6], we denote by $\mathcal{I}_{u}(G)$ the subset of $\mathcal{I}(G)$ consisting of the uniformly fully inert subgroups, that is, of the subgroups $H$ such that $|(\phi H+H) / H| \leq N$ for all endomorphisms $\phi$ and for a fixed positive integer $N$. In [6] it was proved that $\operatorname{Inv}(G) \subseteq$ $\mathcal{I}_{u}(G)$. We call $\mathcal{I}_{u}(G)$ the uniform full inertia set of $G$.

Since this paper offers several new examples of fully inert subgroups of $p$-groups not commensurable with fully invariant subgroups, our goal is to show that also these examples are not uniformly fully inert, thus strengthening the conjecture in [6], namely, that $\operatorname{In} \tilde{v^{\sim}}(G)=$ $\mathcal{I}_{u}(G)$ for all groups $G$. More precisely, we would like to prove that the fully inert subgroups exhibited in Theorem 2.4, Proposition 3.7 and Proposition 3.9 are not uniformly fully inert. The next three results provide these desired proofs.

We prove the first result for the fully inert subgroup $H^{F}[p]$ of $G$, and we leave to the reader to extend the result to higher socles $H^{F}\left[p^{k}\right]$, for $k>1$.

Proposition 5.1 The fully inert subgroup $H^{F}[p]$ of $G$ of Theorem 2.4 is not uniformly fully inert.

Proof We must show that for every positive integer $k$ there exists an endomorphism $\phi \in$ End $(G)$ such that $|(\phi K+K) / K| \geq k$, where $K=H^{F}[p]$. We have seen in the proof of Theorem 2.4 that $K$ is contained in a countable pure subgroup $C=\bigoplus_{i \in \mathbb{N}} C_{i}$, where each $C_{i}$ is a finite direct sum of cyclic groups isomorphic to $\mathbb{Z}\left(p^{i}\right)$. As $K$ is infinite, there exists a positive integer $n$ such that $\left|K \cap \bigoplus_{1 \leq i \leq n} C_{i}\right| \geq k$. Note that $\bigoplus_{1 \leq i \leq n} C_{i}$ is a direct summand of $G$. We shall find an embedding $\phi: \bigoplus_{1 \leq i \leq n} C_{i} \rightarrow G$ such that $\operatorname{Im}(\phi) \cap C=0$. Extend $\phi$ to an endomorphism of $G$, still called $\phi$, which sends a complement of $\bigoplus_{1 \leq i \leq n} C_{i}$ to 0 . In such a way we have that $\phi K$ which contains $\phi\left(K \cap \bigoplus_{1 \leq i \leq n} C_{i}\right)$, has cardinality at least $k$ and $(\phi K+K) / K \cong \phi K$, because $\operatorname{Im}(\phi) \cap C=0$ implies $\phi K \cap K=0$; so our claim will follow.

In order to define the map $\phi$, we make use of an idea used in the proof of [6, Lemma 6.1]. By a classical result by Kovács (see [11, Theorem 5.12]), the subgroup $C$ is contained in a basic subgroup $B$ of $G$. Since $G$ is uncountable, by Lemma 2.3 , and $B$ is countable since $G$ is semi-standard, we have that the divisible group $G / B$ has uncountable rank, therefore it contains a subgroup $A / B$ isomorphic to $C$. But $C$, being a direct sum of cyclic groups, is pure-projective, hence we have a direct decomposition $A=B \oplus C^{\prime}$, with $C^{\prime} \cong C$; therefore 
$B \cap C^{\prime}=0$ and consequently also $C \cap C^{\prime}=0$. Now the desired map $\phi: \bigoplus_{1 \leq i \leq n} C_{i} \rightarrow G$ is the restriction to $\bigoplus_{1 \leq i \leq n} C_{i}$ of the isomorphism $C \cong C^{\prime}$. Thus, we are done.

The second result makes use of an idea used in the proof of [13, Lemma 3.2].

Proposition 5.2 The fully inert subgroup $H=A[p] \oplus\{0\}$ of $G=A \oplus C$ of Proposition 3.7 is not uniformly fully inert.

Proof We must show that for every positive integer $k$ there exists an endomorphism $\phi \in$ $\operatorname{End}(A \oplus C)$ such that $|(\phi A[p]+A[p]) / A[p]| \geq k$.

Let $B=\bigoplus_{i \in I}\left\langle e_{i}\right\rangle$ be a basic subgroup of $A$ and $B^{\prime}=\bigoplus_{j \in J}\left\langle e_{j}^{\prime}\right\rangle$ a basic subgroup of $C$. Select a sequence of cyclic summands $\left\langle e_{i_{n}}\right\rangle(n \in \mathbb{N})$ of $B$ and a sequence of cyclic summands $\left\langle e_{j_{n}}^{\prime}\right\rangle(n \in \mathbb{N})$ of $B^{\prime}$ of strictly increasing order, such that the order of $e_{j_{n}}^{\prime}$ is greater than or equal to the order of $e_{i_{n}}$. Then define a map $\sigma: B \rightarrow B^{\prime}$ by embedding each $e_{i_{n}}$ into $e_{j_{n}}^{\prime}$, and sending to zero all the remaining generators $e_{i}$.

Choose now an index $N$ such that $\left|\left(\bigoplus_{1 \leq n \leq N}\left\langle e_{i_{n}}\right\rangle\right)[p]\right| \geq k$ and define the endomorphism $\phi$ of $G=A \oplus C$ in the following way: $\phi C=0, \phi\left(\bigoplus_{1 \leq n \leq N}\left\langle e_{i_{n}}\right\rangle\right)=\sigma\left(\bigoplus_{1 \leq n \leq N}\left\langle e_{i_{n}}\right\rangle\right)$ and $\phi$ vanishes on a complement of $\bigoplus_{1 \leq n \leq N}\left\langle e_{i_{n}}\right\rangle$ in $A$. It is clear that $|(\phi A[p]+A[p]) / A[p]| \geq k$, since $(\phi A[p]+A[p]) / A[p] \cong \sigma\left(\left(\bigoplus_{1 \leq n \leq N}\left\langle e_{i_{n}}\right\rangle\right)[p]\right)$.

The proof of the last result makes use of arguments similar to those used in Propositions 5.1 and 5.2, taking care that the groups $G_{\sigma}$ have the same basic subgroup $B=\bigoplus_{n} \mathbb{Z}\left(p^{n}\right)$, and that the subgroup $K_{\tau}$ is infinite. We just sketch the proof and leave the details to the reader.

Proposition 5.3 The fully inert subgroup $K$ of $G=\bigoplus_{\sigma \in \Sigma} G_{\sigma}$ of Proposition 3.9 is not uniformly fully inert.

Proof We must show that for every positive integer $k$ there exists an endomorphism $\phi \in$ $\operatorname{End}(G)$ such that $|(\phi(K)+K) / K| \geq k$. We use the notation of Proposition 3.9: so $K$ is the direct sum of a single subgroup $K_{\tau}$ and the zero subgroups of all the $G_{\rho}$ with $\rho \in \Sigma$ and $\rho \neq \tau$. Now it follows from the proof of Proposition 5.1 that, for any given positive integer $k$, there is an endomorphism, $\phi_{\tau}$ say, of $G_{\tau}$ such that $\left|\left(\phi_{\tau}(K)+K_{\tau}\right) / K_{\tau}\right| \geq k$. Extend $\phi_{\tau}$ to an endomorphism $\phi$ of $G$ by setting $\phi \uparrow G_{\tau}=\phi_{\tau}$ and setting $\phi$ to be identically zero on the complement $\bigoplus_{\rho \neq \tau} G_{\rho}$ of $G_{\tau}$ in $G$. It follows immediately that $|(\phi(K)+K) / K|=$ $\left|\left(\phi_{\tau}\left(K_{\tau}\right)+K_{\tau}\right) / K_{\tau}\right| \geq k$.

Question 7.5 in [6] asks whether the $p$-group $G$ of Theorem 2.4 satisfies the equality $\mathcal{I}_{n} \tilde{v^{(}(G)}=\mathcal{I}_{u}(G)$. In the same way we may ask whether this equality holds also for the groups considered in Proposition 3.7 and Proposition 3.9.

Funding Open access funding provided by Università degli Studi di Padova within the CRUI-CARE Agreement.

Open Access This article is licensed under a Creative Commons Attribution 4.0 International License, which permits use, sharing, adaptation, distribution and reproduction in any medium or format, as long as you give appropriate credit to the original author(s) and the source, provide a link to the Creative Commons licence, and indicate if changes were made. The images or other third party material in this article are included in the article's Creative Commons licence, unless indicated otherwise in a credit line to the material. If material is not included in the article's Creative Commons licence and your intended use is not permitted by statutory regulation or exceeds the permitted use, you will need to obtain permission directly from the copyright holder. To view a copy of this licence, visit http://creativecommons.org/licenses/by/4.0/. 


\section{References}

1. G. Braun, L. Strüngmann, The independence of the notions of Hopfian and co-Hopfian Abelian p-groups. Proc. Am. Math. Soc. 143, 3331-3341 (2015)

2. A.R. Chekhlov, On fully inert subgroups of completely decomposable groups. Math. Notes (2) 101, 365-373 (2017)

3. A. R. Chekhlov, P. Danchev, B. Goldsmith, On the socles of fully inert subgroups of abelian $p$-groups, Mediterr. J. Math 18(3), 20 (2001)

4. A.L.S. Corner, On endomorphism rings of primary Abelian groups. Q. J. Math. Oxford 20(2), 277-296 (1969)

5. U. Dardano, D. Dikranjan, S. Rinauro, Inertial properties in groups. Int. J. Group Theory 7(3), 17-62 (2018)

6. U. Dardano, D. Dikranjan, L. Salce, On uniformly fully inert subgroups of abelian groups, Proc. Udine Conf., Topol. Algebra Appl. 8(2020), 5-27 (2018)

7. D. Dikranjan, A. Giordano Bruno, L. Salce, S. Virili, Fully inert subgroups of divisible Abelian groups. J. Group Theory 16, 915-939 (2013)

8. D. Dikranjan, B. Goldsmith, L. Salce, P. Zanardo, Algebraic entropy for abelian groups. Trans. Am. Math. Soc. 361, 3401-3434 (2009)

9. M. Dugas, On the Jacobson radical of some endomorphism rings. Proc. Am. Math. Soc. 102(4), 823-826 (1988)

10. P. Eklof, A. Mekler, Almost Free Modules, Set Theoretic Methods, revised edn. (North-Holland, Amsterdam, 2002)

11. L. Fuchs, Abelian Groups, Springer Monographs in Math (Springer, New York, 2015)

12. R. Göbel, W. May, Modular group algebras of $\aleph_{1}$-separable p-groups. Proc. Am. Math. Soc. 131(10), 2987-2992 (2003)

13. B. Goldsmith, L. Salce, Algebraic entropies for Abelian groups with applications to their endomorphism rings: a survey, in Groups, Modules, and Model Theory-Surveys and Recent Developments (Springer, 2017) pp. 135-175

14. B. Goldsmith, L. Salce, Fully inert subgroups of torsion-complete p-groups. J. Algebra 555, 406-424 (2020)

15. B. Goldsmith, L. Salce, P. Zanardo, Fully inert subgroups of Abelian p-groups. J. Algebra 419, 332-349 (2014)

16. P. Hill, On the decomposition of groups. Can. J. Math. 21, 762-768 (1969)

17. M. Huber, Methods of set theory and the abundance of separable abelian p-groups, Lecture Notes Math. N. 1006 (Springer, Berlin, 1983), pp. 304-319

18. P. Keef, Countably totally projective $p$-groups have minimal full inertia. J. Commut. Algebra (to appear)

19. C. Megibben, $\omega_{1}$-separable $p$-groups, Abelian Group Theory (Oberwolfach, 1985) (Gordon and Breach, New York, 1987), pp. 117-136

20. R.S. Pierce, Homomorphisms of Primary Abelian Groups, Topics in Abelian Groups (Illinois, Chicago, 1963), pp. 215-310

21. L. Salce, La struttura dei p-gruppi abeliani, Quaderni dell’U.M.I. n. 18, Pitagora Ed., Bologna (1980)

22. L. Salce, P. Zanardo, Commutativity modulo small endomorphisms and endomorphisms of zero algebraic entropy, in Models, Modules and Abelian Groups (de Gruyter 2008), pp. 487-497

23. A.D. Sands, On the radical of the endomorphism ring of a primary abelian group, in Abelian Groups and Modules, Proc. Udine Conference 1984 (Springer, 1984), pp. 304-314

Publisher's Note Springer Nature remains neutral with regard to jurisdictional claims in published maps and institutional affiliations. 